Case report

\title{
APPLICATION OF PLATELET-RICH PLASMA FOR CANINE OSTEOARTHRITIS TREATMENT - A CLINICAL SERIES
}

\author{
K. B. AMINKOV, N. H. MEHANDZHIYSKI, B. Y. AMINKOV \\ \& N. Z. ZLATEVA-PANAYOTOVA \\ Department of Surgery, Radiology, Anesthesiology, Obstetrics and Gynecology, \\ Faculty of Veterinary Medicine, University of Forestry, Sofia, Bulgaria
}

\section{Summary}

Aminkov, K. B., N. H. Mehandzhiyski, B. Y. Aminkov \& N. Z. Zlateva-Panayotova, 2021. Application of platelet-rich plasma for canine osteoarthritis treatment - a clinical series. Bulg. J. Vet. Med., 24, No 4, 601-607.

Osteoarthritis, also known as degenerative joint disease (DJD), is defined as a progressive and permanent long-term deterioration of the cartilage surrounding the joints. There is no known cause for primary DJD. However, there are a wide variety of causes for secondary DJD, such as trauma, abnormal wear of joints and cartilage, or a congenital defect present at birth such as an improperly formed hip. One of the most popular methods used to biologically enhance healing in the fields of orthopaedic surgery and medicine includes the use of autologous blood products, namely, platelet rich plasma (PRP). Reports suggest that PRP, presumably containing high levels of platelet growth factors, may promote the recovery of the affected cartilage. This case series presents clinical and radiographic findings of three dogs with osteoarthritis of the elbow and knee joints. Pain score were assessed by CBPI (Canine Brief Pain Inventory). Treatment with three-fold intra-articular application of PRP, obtained by double centrifugation method, resulted in significant improvement in the function of the affected joint. Therefore, it could be concluded that PRP was clinically effective in the treatment of osteoarthritis in these three cases.

Key words: dog, osteoarthritis, platelet-rich plasma

Osteoarthritis $(\mathrm{OA})$ is a complex disease affecting all joint structures. Usually, it is diagnosed in its late, irreversible stages. Until now, there is no known disease modifying therapy (DMT) to effectively modify the disease and prevent its progression (Mobasheri \& Henrotin, 2010). Currently, there is ongoing research on possible alternative treatment approaches such as cytokine inhibitors, genetic therapy and the use of growth factors which could potentially protect the normal joint homeostasis or reverse the structural changes in joints affected by OA (Filardo et al., 2012; Spakova et al., 2012). Usually, OA therapy is aimed at providing 
pain and inflammation relief (Mobasheri \& Henrotin, 2010), maintaining joint motility in order to improve the patient's quality of life and delay disease progression (Gigante \& Callegari, 2011). Nonsteroidal anti-inflammatory drugs (NSAIDs) are amongst the most frequently used therapies in the dog. Prolonged use of NSAIDs provides good pain control and joint mobility (Innes et al., 2010).

Other commonly used agents for intraarticular therapy are corticosteroids. Although their exact mechanism of action still remains unclear, their effect is believed to be mediated by inhibiting phospholipase activity which results in reduced production of cyclooxygenases and lipoxygenases (Schulz, 2007). These reduce the number of inflammatory cells, such as lymphocytes, macrophages and mast cells, and thus reduce phagocytosis, the release of lysosomal enzymes and inflammatory mediators (Lavelle et al., 2007).

Hyaluronic acid is administered intraarticularly to improve the viscosity and elasticity of the synovial fluid and inhibit the pain associated with joint movement. In vitro studies suggest that hyaluronic acid has anti-inflammatory, anti-septic and chondroprotective effects (Edwards, 2011; Colen et al., 2012).

The application of platelet-rich plasma (PRP) has also been recently introduced in the clinical practice. Apart being involved in the blood clotting pathway, platelets also contain growth factors and cytokines that are essential for soft tissue healing and bone mineralisation. Many of the bioactive proteins released from the platelets resemble those secreted by macrophages, mesenchymal stem cells and osteoblasts, and thus play an essential role in tissue regeneration and healing (Sampson et al., 2010). Growth factors contained in platelets such as platelet- derived growth factor (PDGF), transforming growth factor 1 (TGF-1), insulin-like growth factor 1 (IGF-1), vascular endothelial growth factor (VEGF), basic fibroplast growth factor (bFGF) and epidermal growth factor (EGF), have been shown to take part in the regulation and synthesis of articular cartilage. These are also a source of cytokines, chemokines and other proteins that stimulate cell proliferation, chemotaxis and modulate inflammatory response (Kon et al., 2011). It has been shown that high platelet concentrations will deliver high concentrations of the aforementioned growth factors and thus activate and stimulate the healing processes that normally occur following trauma (Sampson et al., 2010; Stief et al., 2011; Spakova et al., 2012).

Intra-articular administration of platelet-rich plasma (PRP) can slow the progression of osteoarthritis by stimulating cartilage anabolism (Stief et al., 2011). As PRP is an autologous blood product, there is no risk of immunological reactions and disease transfer, but as with any injection procedure, there will be some possibility of a local anaesthesia reaction, infection and bleeding (Sampson et al., 2008). PRP contains a high concentration of platelets that, once activated, release numerous growth factors into the surrounding environment. The resulting cellular effects are both pro- and anti-inflammatory in nature and appear to be dependent on different factors including the stage of the natural healing process, the site of the injury, and cellular environment (Middleton et al., 2012).

The purpose of the study was to investigate the effectiveness of platelet-rich plasma (PRP) in dogs with osteoarthropathies of the elbow and knee joints. In particular, our aim was to evaluate the magnitude of clinical improvement after a 
triple intra-articular injection of an autologous platelet concentrate in dogs with osteoarthritis.

\section{Case series}

The current study was conducted in Veterinary Clinic "AMI VET" in Sofia for the period 2016-2017. Three dogs with osteoarthritis of the elbow joint and osteoarthritis of the knee joint were included in the study.

Patient 1 was a 7-year-old male American Bulldog, weighing $55 \mathrm{~kg}$. It was referred to the clinic due to the development of high-grade lameness of the right forelimb, swelling and severe pain upon palpation of the elbow joint. Lameness had occurred about a month ago, and a week before the dog was brought to the clinic, it was treated with an antibiotic. The dog was tested for the following vector-borne infections: anaplasmosis, Lyme disease, ehrlichiosis, leishmaniasis; test results were negative.

Synovial fluid was collected from the affected joint by arthrocentesis and was sent for microbiological examination and antibiogram. Synovial fluid was haemorrhagic. The result from the microbiological examination for the presence of Gram negative and Gram positive bacteria was negative. Advanced osteoarthritis of the elbow joint was radiologically confirmed.

Patient 2 was a 9-year-old German Shepherd dog, weighing $34 \mathrm{~kg}$. Eighteen months ago, during active movement, lameness appeared in the left hindlimb. Since 25 December 2016, severe lameness has occurred and the dog was reluctant to put weight on the leg. From 16 to 22 January 2017, the dog was treated with NSAIDs (PREVICOX®), with no effect from the therapy. The dog was tested for anaplasmosis, Lyme disease, ehrlichosis, leishmaniasis. Test results were negative.
Clinical examination revealed highgrade lameness in the left hindlimb resulting in reluctance to put weight on the limb. There was severe pain, as well as difficulty standing up. Advanced osteoarthritis of the knee joint was radiologically confirmed.

Patient 3 was a 3-year-old English Bulldog with bilateral dysplasia of the elbow joints - fragmented coronoid process (FCP). The patient has undergone surgical interventions to treat the problem one year after severe lameness has appeared and was reluctant to move. Test results for anaplasmosis, Lyme disease, erlichiosis and leishmaniasis was negative. Advanced osteoarthritis of the elbow joints was radiologically confirmed.

\section{Anaesthetic protocol}

PRP application was performed under general anaesthesia with the following anaesthetic protocol: premedication with $\mathrm{SC}$ administration of $0.02 \mathrm{mg} / \mathrm{kg}$ atropine sulphate, followed by IM administration of $0.8 \mathrm{mg} / \mathrm{kg}$ xylazine hydrochloride after $15 \mathrm{~min}$. Induction was performed using 5 $\mathrm{mg} / \mathrm{kg}$ propofol. Maintenance of anaesthesia was achieved using $0.04 \mathrm{mg} / \mathrm{kg} / \mathrm{min}$ propofol.

\section{Assessment of pain severity and pain interference score}

At day 0 and 10 days after the last intraarticular PRP treatement, pain scores were assigned by owners of the dogs on the basis of the Canine Brief Pain Inventory (CBPI) (Brown et al., 2008). The principle of CBPI evaluation was explained in detail to dog owners. The standard CBPI contains four items pertaining to the severity of the dog's pain and six items describing how that pain interferes with the dog's daily activities, and uses lower 
numbers to indicate less severe pain $(0=$ no pain; 10 = worst pain).

\section{PRP preparation}

Sixteen millilitres of citrated blood were centrifuged (Labofuge 400, Heraeus Holding, Hanau, Germany) without applying a brake for $20 \mathrm{~min}$ at $2800 \mathrm{rpm}$ to achieve separation of cell layers. This procedure divides the blood into three basic components: red blood cells, platelet rich plasma (PRP) and platelet poor plasma (PPP). Red blood cells were isolated from the overlying buffy coat and plasma by the gel-like plug within the tubes. Each tube ( $8 \mathrm{~mL}$ of blood) thus yielded approximately $4-5 \mathrm{~mL}$ of platelet-poor plasma, of which $80 \%$ was discarded. The buffy coat of each tube, containing mononuclear cells and platelets, was then carefully removed with a pipette and resuspended in $0.75-1.0 \mathrm{~mL}$ of the plasma remaining. The final solution, obtained by mixing different buffy coats in a sterile $15 \mathrm{~mL}$ Falcon tube, was centrifuged without applying a brake at $1300 \mathrm{rpm}$ for $15 \mathrm{~min}$ for good separation of platelet pellets in the supernatant layer. The platelet pellet accumulates at the bottom of the tube, the PPP on top. The PPP is drawn off so that the PRP remains in the tube. After resuspending the platelet pellet within the remaining volume of plasma with the vortex mixer, the final PRP can be drawn up with a syringe. Each step of the method was carried out using sterile disposables (Perazzi et al., 2013). No topical or systemic drugs, antibiotics or anti-inflammatory drugs were administered to dogs during the PRP treatment.

In Patient 1, intra-articular injection of platelet-rich plasma (PRP) was performed three times every 10 days. Ten days after the first treatment significant reduction in lameness was observed, as well as signifi- cant reduction of swelling in the area of the elbow joint and improvement of joint mobility. Synovial fluid was yellowish. After the second treatment, minimal lameness, no swelling or pain were observed in the area of the affected joint. Following the third therapy there was no lameness at all. The period of improvement lasted for 6 months.

In patient 2, intra-articular injection of platelet-rich plasma (PRP) was performed three times every 10 days. Ten days after the first treatment the dog started to put weight on the affected limb, and to use it when standing up. Mild lameness was observed. After the second and third treatments lameness was significantly reduced. The period of improvement lasted for 6 months.

In patient 3 , the intra-articular injection of platelet-rich plasma (PRP) was performed in both elbow joints three times every 10 days. After the treatment, lameness was significantly reduced and the period of improvement lasted for 8 months. Another treatment was performed after that. The period of improvement lasted for 8 months

Pain severity and pain interference score assigned by owners of the dogs using the CBPI are presented in Table 1.

In clinical practice, PRP is administered with either thrombin or calcium chloride activated before or inactivated. Seventy percent of the growth factors in alpha-granules are released after $10 \mathrm{mi}$ nutes and almost all in one hour (WangSaegusa et al., 2011). It was found that without pro-activator, pure PRPs are activated slowly upon contact with collagen I (Mishra et al., 2009). In our study, we used nonactivated PRP. In vivo studies show that non-activated PRP stimulates chondrogenesis at day 14 and osteogenesis of 28 and 56 day as activated throm- 
Table 1. Pain severity and pain interference score assigned by owners of the dogs at day 0 and 10 days after the last intraarticular PRP treatement using Canine Brief Pain Inventory

\begin{tabular}{lcccccc}
\hline \multirow{2}{*}{ Variable: } & \multicolumn{2}{c}{ Patient 1 } & \multicolumn{2}{c}{ Patient 2 } & \multicolumn{2}{c}{ Patient 3 } \\
\cline { 2 - 7 } & Before & After & Before & After & Before & After \\
\hline Worst pain & 7 & 2 & 7 & 3 & 6 & 2 \\
Least pain & 3 & 1 & 4 & 3 & 4 & 2 \\
Typical pain & 4 & 2 & 5 & 2 & 5 & 3 \\
Pain now & 4 & 2 & 4 & 2 & 4 & 1 \\
General activity & 5 & 1 & 4 & 3 & 5 & 1 \\
Enjoys life & 3 & 2 & 4 & 2 & 5 & 1 \\
Can rise & 7 & 3 & 6 & 3 & 6 & 2 \\
Can walk & 4 & 1 & 5 & 2 & 6 & 2 \\
Can run & 4 & 1 & 5 & 2 & 4 & 3 \\
Can climb & 3 & 3 & 6 & 3 & 3 & 3 \\
& 4.4 & 1.8 & 5.0 & 2.5 & 4.8 & 2.0 \\
\hline
\end{tabular}

CBPI results at day 10 showed significant improvement in all pain severity and pain interference score. Mean point improvement varied between 2.5 and 2.8, with all three patients.

bin-PRP acts to inhibit these processes (Han et al., 2009). In our study there was an effect after the triple application of PRP at 10-day intervals. After the second and third treatment lameness was significantly reduced. Lameness and pain scores according to CBPI system were significantly improved. The owners reported significant improvement in quality of life of the pets including lameness, pain walking, running, and enjoying life. The average duration of observed improvement lasted about 6 to 8 months.

Sampson et al. (2010) achieved significant pain relief and articular joint repair in the area of the lateral and medial condyles of the femur following application of PRP three times every other month.

Milano et al. (2010) have studied the effect of intra-articular administration of PRP in sheep with complete articular cartilage damage and found significant tissue recovery compared to the control group using histological and macroscopic evaluation.
Some of the advantages of autologous PRP are lack of significant side effects, immune response or transmission of infectious diseases (Cole et al., 2010; Filardo et al., 2012). It was reported that intraarticular injection of PRP not only affects articular cartilage but also all joint structures and other cell sources. Furthermore it plays a complex role in the mechanisms of inflammation. In addition to all these mechanisms, PRP has a direct analgesic effect (Lee et al., 2016).

In conclusion, platelet-rich plasma shows a promising and significant therapeutic potential in the treatment of osteoarthropathies of the elbow and knee in large and giant dog breeds. Regenerative therapy can be used to relieve pain, improve mobility of the damaged joint, and slow down the development of clinical signs of osteoarthritis in the dog. After a triple intraarticular injection of plateletrich plasma, clinical improvement in patients with osteoarthritis of the knee or elbow joint lasted between 6 and 8 
months. The limitation of the study is related to the low number of discussed clinical cases, so further investigations are required to draw valuable conclusions and validate the findings.

\section{REFERENCES}

Brown, D. C., R. C. Boston, J. C. Coyne \& J. T. Farrar, 2008. Ability of the canine brief pain inventory to detect response to treatment in dogs with osteoarthritis. Journal of the American Veterinary Medical Association, 233, 1278-1283.

Cole, J., T. Seroyer, S. Bajaj \& A. Fortier, 2010. Platelet-rich plasma: Where are we now and where are we going? Sports Health: Multidisciplinary Approach, 3, 203-210.

Colen, S., M. Van Den Bekerom, M. Mulier \& D. Havenkamp, 2012. Hyaluronic acid in the treatment of knee osteoarthritis: systematic review and meta-analysis with emphasis on the efficacy of different products. BioDrugs: Clinical Immunotherapeutics, Biopharmaceuticals and Gene Therapy, 26, 257-268.

Cook, J. L., C. C. Anderson, J. M. Kreeger \& J. L.Tomlinson, 2000. Effects of human recombinant interleukin-1 beta on canine articular chondrocytes in three-dimensional culture. American Journal of Veterinary Research, 61, 766-776.

Edwards, R., 2011. Intra-articular drug delivery: The challenge to extend drug residence time within the joint. The Veterinary Journal, 190, 15-21.

Everts, P. A., M. M. Hoogbergen, T. A. Weber, R. J. Devilee, G. van Monftort \& I. H. de Hingh, 2012. Is the use of autologous platelet-rich plasma gels in gynecologic, cardiac, and general, reconstructive surgery beneficial? Current Pharmaceutical Biotechnology, 13, 1163-1172.

Filardo, G., E. Kon, P. Ruiz, F. Vaccaro, R. Guitaldi, D. Martino, A. Cenacchi, M. Fornasari \& M. Marcacci, 2012. Platelet- rich plasma intra-articular injections for cartilage degeneration and osteoarthritis: Single-versus double-spinning approach. Knee Surgery, Sports Traumatology, Arthroscopy, 20, 2082-2091.

Gigante, A. \& L. Callegari, 2011. The role of intra-articular hyaluronan (Sinovial ${ }^{\circledR}$ ) in the treatment of osteoarthritis. Rheumatology International, 31, 427-444.

Han, B., J. Woodell-May, M. Ponticiello, Z. Yang \& M. Nimni, 2009. The effect of thrombin activation of platelet-rich plasma on demineralized bone matrix osteoinductivity. Journal of Bone and Joint Surgery, 9, 1459-1470.

Innes, F., J. Glayton \& D. Lascelles, 2010. Review of the safety and efficacy of longterm NSAID use in the treatment of canine osteoarthritis. The Veterinary Record, 166, 226-230.

Kon, E., G. Filardo, A. Martino \& M. Marcacci, 2011. Platelet-rich plasma (PRP) to treat sports injuries: Evidence to support its use. Knee Surgery, Sports traumatology, Arthroscopy, 19, 516-527.

Lavelle, W., E. Lavelle Demers \& L. Lavelle, 2007. Intra-articular injections. Anesthesiology Clinics, 25, 853-862.

Lee, H. R., O. J. Shon, S. I. Park, H. J. Kim, S. Kim, M. W. Ahn \& S. H. Do, 2016. Platelet-rich plasma increases the levels of catabolic molecules and cellular dedifferentiation in the meniscus of a rabbit model. International Journal of Molecular Sciences, 17, 120-127.

Middleton, K. K., V. Barro, B. Muller, S. Terada, \& F. H. Fu, 2012. Evaluation of the effects of platelet-rich plasma (PRP) therapy involved in the healing of sportsrelated soft tissue injuries. Iowa Orthopedic Journal, 32, 150-163.

Milano, G., E. Sanna Passino, L. Deriu, G. Careddu, L. Manunta, A. Manunta, M. Saccomanno \& C. Fabbriciani, 2010.The effect of platelet rich plasma combined with microfractures on the treatment of chondral defects: An experimental study in 
sheep model. Osteoarthritis and Cartilage, 18, 971-980.

Mishra, A., J. Jr. Woodall \& A. Vieira, 2009. Treatment of tendon and muscle using platelet-rich plasma. Clinics in Sports Medicine, 28, 113-125.

Mobasheri, A. \& Y. Henrotin, 2010. Identification, validation and qualification of biomarkers for osteoarthritis in humans and companion animals: Mission for the next decade. The Veterinary Journal, $\mathbf{1 8 5}$, 95-97.

Perazzi, A., R. Busetto, T. Martinello, M. Drigo, D. Pasotto, F. Cian, M. Patruno \& I. Iacopetti, 2013. Description of a double centrifugation tube method for concentrating canine platelets. BMC Veterinary Research, 9, 146-155.

Sampson, S., M. Reed, H. Silvers, M. Meng \& B. Mandelbaum, 2010. Injection of platelet rich plasma in patients with primary and secondary knee osteoarthritis: Pilot study. American Journal of Physical Medicine Rehabilitation, 89, 961-969.

Sampson, S., M. Gerhardt \& B. Mandelbaum, 2008. Platelet rich plasma injection grafts for musculoskeletal injuries: A review Current Reviews in Musculoskeletal Medicine, 1, 165.

Sánchez, M., J. Azofra, E. Anitua, I. Andía, S. Padilla \& J. Santisteban, 2003. Plasma rich in growth factors to treat an articular cartilage avulsion: A case report. Medicine \& Science in Sports \& Exercise, 35, $1648-1652$.

Schulz, K., 2007. Diseases of the joints. In: Small Animal Surgery, $3^{\text {rd }}$ edn, ed T. W. Fossum, Mosby Elsevier, St. Louis, pp. 1143-1315.
Spakova, T., J. Rosocha, D. Harvanova \& A. Gharaibeh, 2012. Treatment of knee joint osteoarthritis with autologous platelet-rich plasma in comparison with hyaluronic acid. American Journal of Physical Medicine Rehabilitation, 91, 411-417.

Stief, M., J. Gottschalk, J. C. Ionita, A. Einspanier, G. Oechtering \& P. Böttcher, 2011. Concentration of platelets and growth factors in canine autologous conditioned plasma. Veterinary and Comparative Orthopaedics and Traumatology, 24, 122-125.

Wang-Saegusa, A., R. Cugat, O. Ares, R. Seijas, X. Cuscó \& M. Garcia-Balletbó, 2011. Infiltration of plasma rich in growth factors for osteoarthritis of the knee shortterm effects on functionn and quality of life. Archives of Orthopaedic and Trauma Surgery, 131, 311-317.

Paper received 18.06.2019; accepted for publication 11.01.2020

\section{Correspondence:}

Dr. N. Mehandzhiyski

Department of Surgery, Radiology,

Anesthesiology, Obstetrics and Gynecology,

Faculty of Veterinary Medicine,

University of Forestry, P.O. Box 1756,

10 "Kliment Ohridsky" str.,

Sofia, Bulgaria

e-mail: nikolay.mehandhiyski@abv.bg 